\title{
Intra-lenticular lens aspiration in paediatric cases with anterior dislocation of lens
}

\author{
Pranita Sahay $^{1} \cdot$ Prafulla K. Maharana $^{1} \cdot$ Nawazish Shaikh $^{1} \cdot$ Siddhi Goel $^{1} \cdot$ Rajesh Sinha $^{1} \cdot$ Tushar Agarwal $^{1}$. \\ Namrata Sharma ${ }^{1} \cdot$ Jeewan S. Titiyal $\mathbb{1}^{1}$
}

Received: 17 December 2018 / Revised: 5 March 2019 / Accepted: 10 March 2019 / Published online: 3 April 2019

(c) The Royal College of Ophthalmologists 2019

\begin{abstract}
Purpose To assess the outcomes of intra-lenticular lens aspiration (ILLA) in paediatric cases with anterior dislocation of lens.

Methods A retrospective review of medical records of cases with anterior dislocation of the lens in children (age $<16$ years) that underwent ILLA between June 2017 and May 2018 was performed. Corrected distance visual acuity (CDVA), intraocular pressure (IOP), and anterior segment findings were noted at presentation and follow-up. Surgical notes were reviewed for all cases. Post-operative central corneal thickness (CCT) and central macular thickness (CMT) were recorded. Results Eleven eyes of eight patients with a median age of ten years underwent ILLA. There were four males and four females. The median duration of symptoms was 2 months, CDVA was $1.77 \log$ MAR, and IOP was $16 \mathrm{~mm}$ of $\mathrm{Hg}$. Ten eyes had corneo-lenticular touch with corneal oedema, and two had raised IOP at presentation. Homocystinuria $(n=2 / 8)$, Microspherophakia $(n=2 / 8)$, Marfan syndrome $(n=1 / 8)$, Buphthalmos $(n=1 / 8)$ and Ectopia lentis et pupillae $(n=1 / 8)$ were the identifiable causes for anterior dislocation. There were no intra-operative complications in any case. Immediate post-operative corneal oedema and raised IOP was observed in nine and three cases respectively and was treated with medical therapy. The median post-operative CDVA and IOP at 6-months was $1 \log \mathrm{MAR}$ and $15 \mathrm{~mm}$ of $\mathrm{Hg}$ respectively. The median CCT and CMT were 516 and $248 \mu \mathrm{m}$ respectively. Five eyes developed a central corneal descemet scar.

Conclusions ILLA is a safe and effective technique for surgical removal of an anteriorly dislocated lens in paediatric cases.
\end{abstract}

\section{Introduction}

Spontaneous anterior dislocation of the crystalline lens in paediatric cases is an ophthalmic emergency that requires immediate surgical intervention. Early surgical intervention is warranted in these cases to avoid long-term blinding complications like corneal decompensation and glaucoma [1]. The management of such cases requires a comprehensive approach as most of these cases are associated with some systemic abnormalities such as Marfan syndrome,

Supplementary information The online version of this article (https:// doi.org/10.1038/s41433-019-0426-y) contains supplementary material, which is available to authorized users.

Prafulla K. Maharana drpraful13@gmail.com

1 Dr Rajendra Prasad Centre for Ophthalmic Sciences, All India Institute of Medical Sciences, New Delhi, India
Weill Marchesini syndrome, Noonan syndrome, Homocystinuria that further complicate the management strategy in these cases [1-4].

The surgical management involves removal of the lens for which various techniques have been described in the literature [1, 2, 4-7]. Herein we describe a modified technique of microscope integrated optical coherence tomography (MiOCT) guided intra-lenticular lens aspiration (ILLA) for management of anteriorly dislocated lens in paediatric cases along with its surgical outcome in perhaps the most extensive series of such cases reported till date.

\section{Methods}

A retrospective review of medical records of all children (age $<16$ years) that presented with anterior dislocation of the lens and underwent ILLA at a tertiary eye care centre between June 2017 and May 2018 were reviewed. The study adhered to the declaration of Helsinki. Approval for 

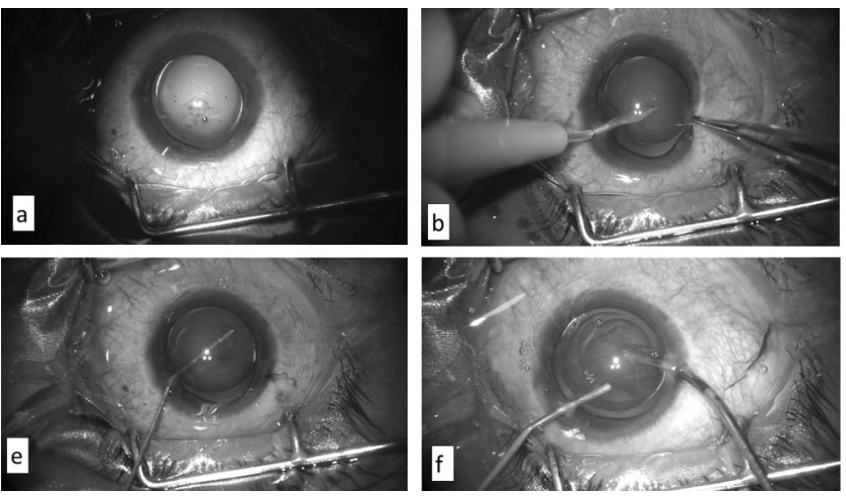

Fig. 1 a Case of anterior dislocation with fixed dilated pupil; $\mathbf{b}$ creation of a clear corneal side port incisions of $0.9 \mathrm{~mm}$ with microvitreoretinal blade at 2 o' clock position with further advancement to make a linear capsulotomy; c creation of a clear corneal side port incisions of $0.9 \mathrm{~mm}$ with microvitreoretinal blade at $10 \mathrm{o}$ clock position with further advancement to make a linear capsulotomy; d

conducting the study was obtained from the institutional review board/ethics committee of All India Institute of Medical Sciences, New Delhi, India.

The medical records of all cases were evaluated for baseline spectacle-corrected distance visual acuity (CDVA), intra-ocular pressure (IOP), anterior segment findings and associated systemic co-morbidities. A careful review of the surgical notes was done to record the various intra-operative difficulties and or complications in all cases. Post-operative CDVA at last follow-up, IOP, anterior segment findings, central corneal thickness (CCT), and central macular thickness (CMT) were noted.

\section{Surgical technique}

Surgery was performed under general anaesthesia in all the cases. The major steps have been depicted in Fig. 1. (Supplementary video). All cases were performed by a single surgeon (PKM). In this procedure two clear corneal side port incisions of $0.9 \mathrm{~mm}$ were made with a $23 \mathrm{~g}$ microvitreoretinal blade (MVR; Alcon Laboratories, Inc.) at 10 and 2 o' clock position (Fig. 1a-c). The MVR was advanced further into the lens capsule to create two linear capsulotomies at the lens equator. Trypan blue [Auroblue $0.6 \%$, Aurolab, India] was injected into the capsulotomies, rather than intracamerally, to stain the margins of the capsular opening (Fig. 1d). This helped in better visualization of the capsulotomy margins while advancing the irrigation and aspiration probe through it into the lens and reduced the risk of inadvertent damage to the lens capsule even with repeated entry of the instruments through the capsulotomy site. A viscodispersive ophthalmic viscoelastic device (OVD) (Viscoat, Alcon Laboratories, Inc., Forth Worth, TX, USA) was injected between the corneal endothelium

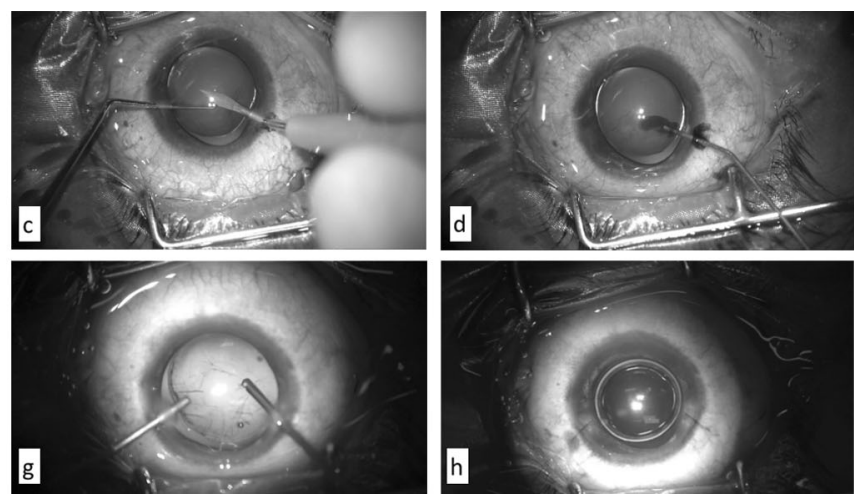

Trypan blue dye injection into the capsulotomies to stain the margins of the capsular opening; e Gentle hydro-dissection and multiple hydrodelineation to create multiple cleavage planes within the crystalline lens; f bimanual irrigation and aspiration within the capsular bag; $\mathbf{g}$ removal of the capsular bag with the help of a vitrectomy cutter

and anterior lens capsule in order to create space between corneal endothelium and anterior capsule of the anteriorly dislocated lens, which were previously stuck to each other. MiOCT was useful at this step as it could delineate the micro-adhesions between the posterior corneal surface and anterior lens capsule accurately (Fig. 2). Pilocarpine was injected below the lens to achieve miosis. Gentle hydrodissection and multiple hydrodelineation were performed to create various cleavage planes within the crystalline lens (Fig. 1e). The irrigation and aspiration cannula was positioned in the bag through the two linear capsulotomies, and lens aspiration was performed within the capsular bag in the anterior chamber (Fig. 1f). The irrigation cannula was used to help in both stabilising the bag during the entire procedure as well as hydrating the lens matter to allow easy aspiration. All these procedures were performed using the Centurion ${ }^{\circledR}$ phacoemulsification system. Although the parameters were variable depending upon the individual cases, overall the IOP was maintained on a lower side $(30-40 \mathrm{~mm}$ of $\mathrm{Hg}$ ) along with a moderately high vacuum $(300-350 \mathrm{~mm}$ of $\mathrm{Hg}$ ) and a low aspiration rate $(24-28 \mathrm{cc} / \mathrm{min})$.

After completion of the lens aspiration, the aspiration cannula was withdrawn, and a vitrectomy cutter was introduced in the capsular bag (Fig. 1g). The capsular bag was then removed with the help of vitrectomy cutter in cut IA mode. Care was taken to remove the anterior capsule first followed by the posterior capsule. This step ensured that there is minimal hydration as well as no traction to the vitreous while removing the capsular remnant. Limited anterior vitrectomy was performed followed by a peripheral iridotomy. At the end of the procedure, the anterior chamber was formed with either a balanced salt solution (BSS) or air. Wherever there was a suspicion of incomplete vitrectomy, $0.1 \mathrm{ml}$ of triamcinolone acetate $(40 \mathrm{mg} / \mathrm{ml}$ ) (Aurocort, Aurolab, India) was injected into the anterior chamber to 
Fig. 2 Micro-adhesions between the posterior corneal surface and anterior lens capsule visible on microscope integrated optical coherence tomography (depicted by red arrows) (colour figure online)

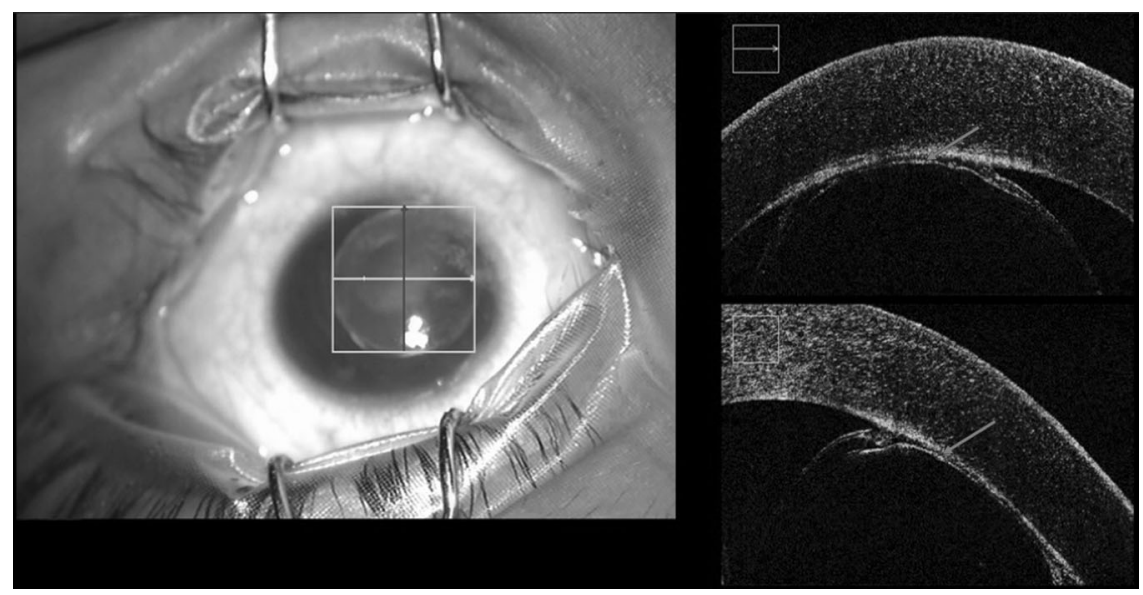

ensure complete vitrectomy. The wound was closed by performing stromal hydration with BSS. A 10-0 monofilament interrupted suture was used for closing the MVR entry whenever it was required (Fig. 1h).

Post-operatively, the patients were prescribed topical moxifloxacin $0.5 \%$ four times a day for 4 weeks, prednisolone phosphate $1 \%$ six times a day for one week followed by tapering over next four weeks and homatropine bromide $2 \%$ four times a day for one week.

\section{Results}

ILLA was performed in 11 eyes of eight patients with spontaneous anterior dislocation of lens (Table 1). The median age was ten years (range, 1.5-14 years) with four males and four females. Three cases presented with bilateral anterior dislocation of the lens. The median duration of presentation from the onset of symptoms was 2 months (range, 3 days to 1 year). The median CDVA at presentation was $1.77 \log$ MAR units (range, 0.78-2.3 $\log$ MAR units) with median IOP of $16 \mathrm{~mm} \mathrm{Hg}$ (range, 4-34 mm Hg). Two cases had raised IOP preoperatively and were managed medically. Ten eyes had corneo-lenticular touch with corneal oedema at presentation. The risk factors for spontaneous anterior dislocation of the lens were Homocystinuria ( $n=2 / 8)$, Microspherophakia $(n=2 / 8)$, Marfan syndrome $(n=1 / 8)$, Buphthalmos $(n=1 / 8)$ and Ectopia lentis et pupillae $(n=1 / 8)$.

ILLA could be performed in all cases without any significant complication. Dense adhesion between the anterior capsule and corneal endothelium was noted in one case, which had to be peeled using intravitreal forceps. All cases were visually rehabilitated with spectacles. Median post-op CDVA at 6-month was 1 logMAR unit with median IOP of $15 \mathrm{~mm}$ of $\mathrm{Hg}$. The median central corneal thickness and central macular thickness were 516 and $248 \mu \mathrm{m}$ respectively. Corneal oedema in the immediate post-operative period was present in nine of eleven eyes that resolved in 1 week. Persistently raised IOP was noted in three eyes that could be successfully managed using anti-glaucoma drugs. Post-operatively five eyes had a central corneal descemet scar. However, none of the cases had corneal decompensation or cystoid macular oedema. No posterior segment complication was noted in any of the cases.

\section{Discussion}

The surgical techniques described in the literature for management of anterior dislocation of the lens include intracapsular cataract extraction (ICCE), lensectomy, and in the bag bimanual lens aspiration [1, 2, 4-7]. Lens aspiration within the capsular bag has been described previously by Vasavada et al., Sinha et al., and Khokhar et al. for various indications [1, 7, 8] (Table 2).

ILLA was performed successfully in all cases with no intra-operative or post-operative complications. It offered several advantages over the previously described surgical techniques. The surgery could be performed without any vitreous prolapse, iris damage, and posterior movement of lens matter during the surgery. Since the whole surgical procedure is performed in a closed chamber compartment, there is reduced risk of complications like vitreous prolapse, supra-choroidal haemorrhage, and significant post-surgery astigmatism, as seen in ICCE [9].

In lensectomy, the lens matter is removed with a vitrectomy cutter. There is always a risk of inadvertent damage to the anterior or posterior capsule with the vitrectomy cutter before complete aspiration of the lens matter, and subsequent lens matter drop into the vitreous cavity. In contrast, ILLA offers a controlled aspiration without the disturbance of vitreous face and nil chances of lens matter drop. Besides, it also prevents excessive vitreous loss during the surgery by avoiding vitreous hydration caused by the irrigation fluid. 


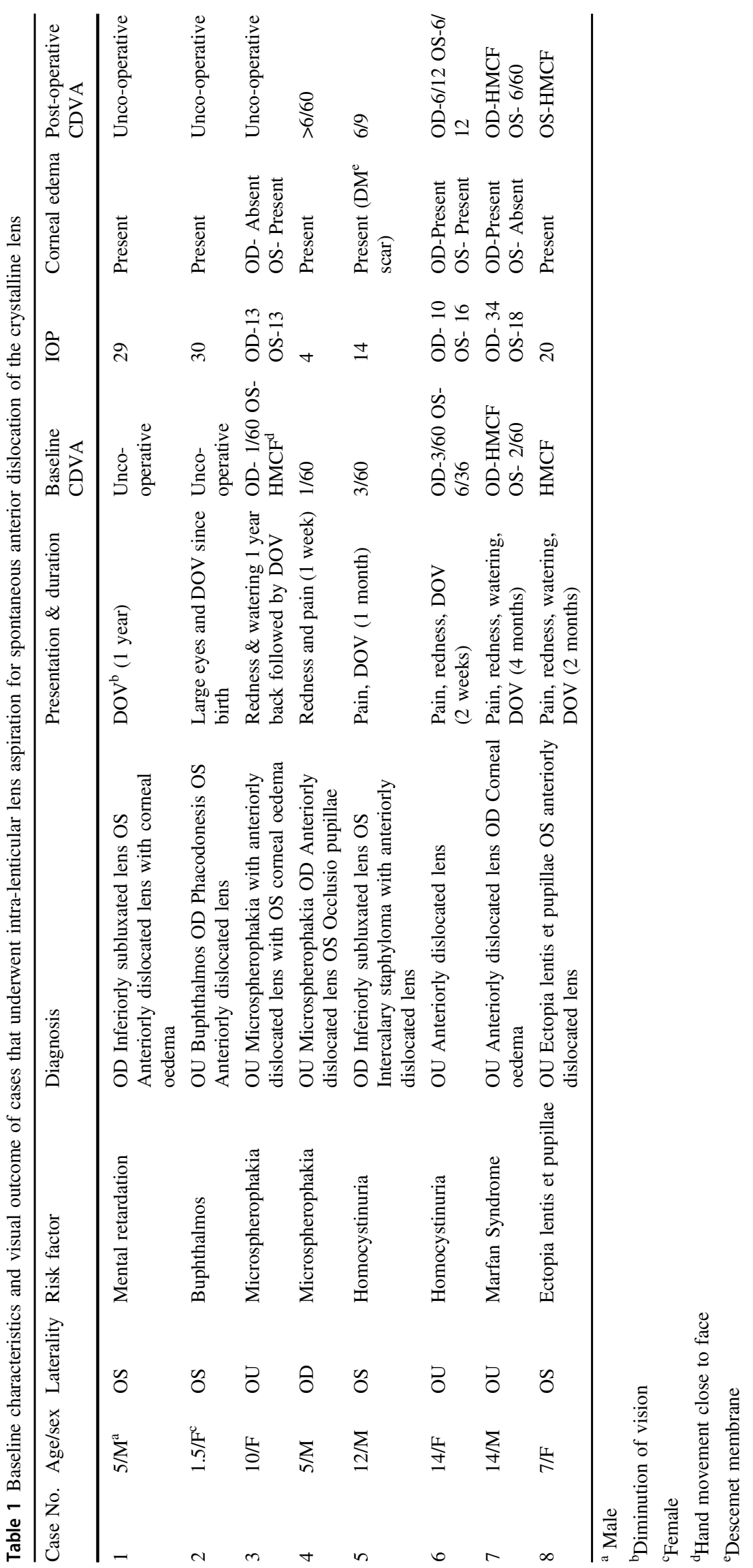




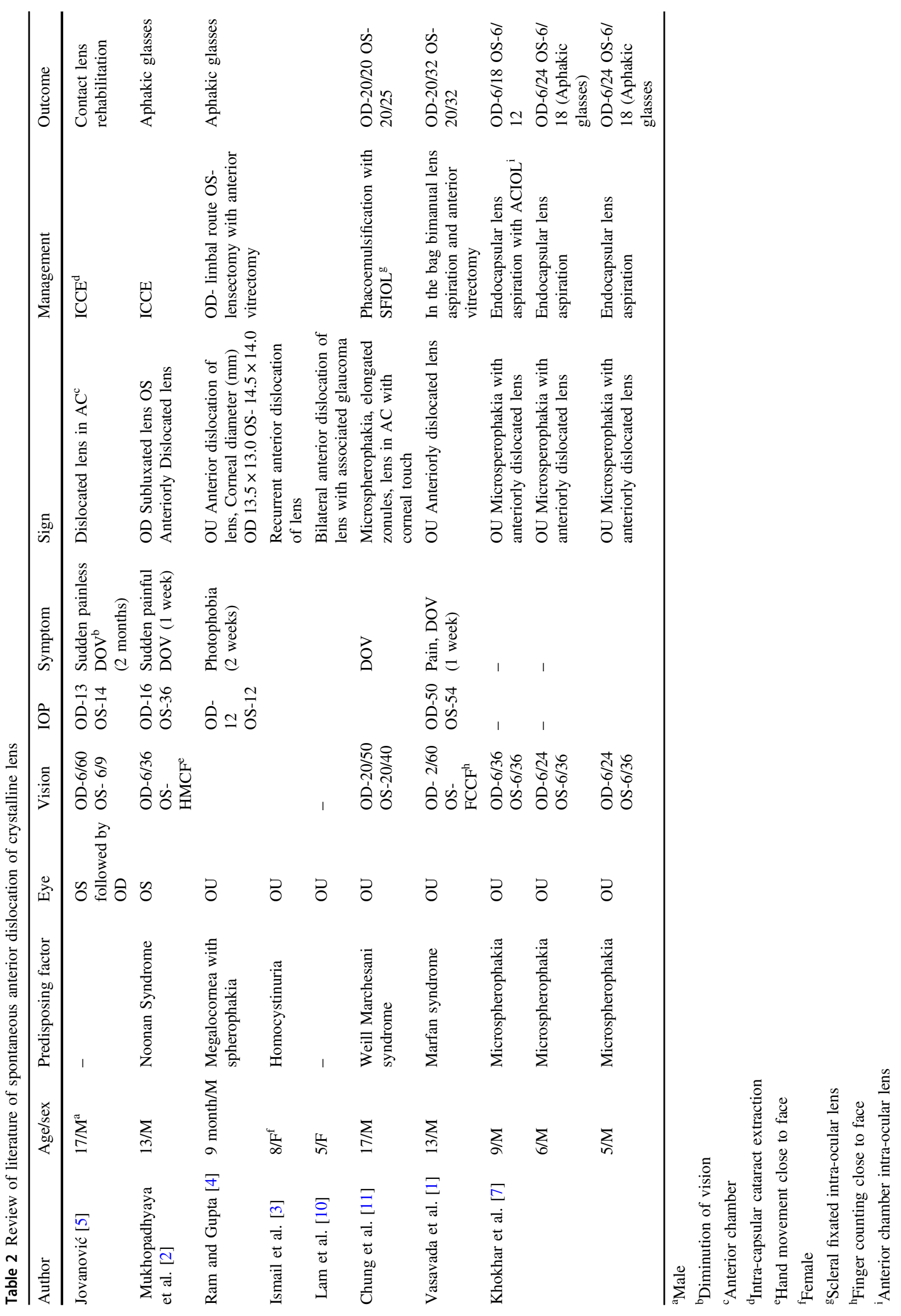


Vasavada et al. [1] in a case report described the surgical removal of an anteriorly dislocated lens in a case of Marfan syndrome by performing in the bag lens aspiration. In this technique, the lens capsule was removed with forceps while severing the capsular adhesions to vitreous with a cutter. This manoeuvre could lead to traction. Hence, we removed the capsule with a vitrectomy cutter. The anterior capsule was removed first followed by posterior to avoid any disturbance to the vitreous interface.

Recently Khokhar et al. [7] described a modified technique of endocapsular lens aspiration for severely subluxated lenses. Our technique differs from this technique in several ways. Khokhar et al. described the technique in cases of lens subluxation while in our series all cases were those of anteriorly dislocated lens with associated corneal oedema. The authors could perform specular microscopy in all their cases while in our series it was not possible due to the associated corneal oedema and photophobia. Thus, our series included cases that were more severe and required immediate surgery, unlike those in Khokhar et al. where the surgery was planned and elective. The second major point of difference, which we believe is the most important, is the use of vitrectomy cutter instead of a bimanual irrigation-aspiration probe by Khokhar et al. Although not ideal, a vitrectomy cutter could be used for aspirating the lens matter. However, the potential risk of inadvertent damage to the lens capsule can't be ruled out completely especially in the presence of corneal oedema and poor visibility, which was present in most of our cases. Damage of anterior lens capsule can increase the chance of lens matter extrusion into the anterior chamber while damage to the posterior capsule could lead to lens matter drop into the vitreous cavity. Once the capsule is damaged the chances of subsequent vitreous hydration and vitreous loss increases significantly. In both, the scenario, the purpose of lens aspiration within the bag is lost. Thus, we believe a bimanual aspiration system without the function of cutting is better in the presence of corneal oedema and poor visibility.

Khokhar et al. [7] did not use trypan blue dye, suggesting that the dye could migrate into the vitreous cavity through the area of zonular dehiscence which could reduce the visibility of the lens and capsular bag on retroillumination. In our technique, we injected trypan blue dye directly into the capsulotomy, instead of intracamerally. This not only made the area of capsulotomy apparent but also prevented any migration posteriorly. In few of our initial cases, we had difficulty in identifying the point of entry while alternating the bimanual probes due to the presence of corneal oedema. Trypan blue staining completely abolished this difficulty. Also, the potential complication of extension of the linear capsulotomies from the inadvertent attempts to enter the instrument in the bag at a wrong position due to poor visualisation of the capsulotomy site could be avoided. Thus, we believe that although the technique described by Khokhar et al. is extremely useful for cases of lens subluxation, our technique may offer several advantages in challenging cases especially those with associated corneal oedema.

Unlike previously described techniques of intracapsular aspiration, we made several modifications to make the surgery safe. We stained the margin of the capsulotomy with trypan blue. In addition to this, multiple rings of hydrodelineation were performed in all the cases. Creating multiple rings of hydro-delineation allowed for easy aspiration of major bulk of the lens matter while the epi-nuclear plate acted as a cushion and protects the lens capsule from inadvertent damage at this step. We removed the lens capsule, anterior followed by posterior, with a vitrectomy cutter.

Out of the 11 surgeries, seven cases were performed under the guidance of MiOCT. We did not find any significant advantage of MiOCT except in two cases, where significant preoperative corneal oedema was making it difficult for us to be $100 \%$ sure about the complete lenticular-corneal adhesion release. Thus, MiOCT may be helpful for cases with poor visibility but not essential.

All of our cases were rehabilitated with spectacles postoperatively. Intra-operatively, we had the option for IOL implantation in the form of intra-scleral fixation of IOL (SFIOL) or anterior chamber IOL (ACIOL). The young age of patients, the presence of corneal oedema, and the risk of pre-existing glaucoma precluded us from considering ACIOL. Most of our cases had systemic associations known to be associated with scleral thinning that discouraged us from considering the option of SFIOL. Contact lens rehabilitation was discussed with the parents postoperatively, but the socioeconomic status and poor compliance were a major concern. Hence all cases were rehabilitated with spectacles.

There are several limitations to our study. The sample size was relatively small which is primarily because of the rarity of the disease. Secondly, the preoperative endothelial cell count could not be measured in any of the cases due to the associated corneal oedema. Thus, we could not determine the endothelial cell loss associated with this technique.

To conclude, our results suggest that Intra-lenticular lens aspiration offers controlled removal of an anteriorly dislocated lens in cases presenting with anterior dislocation of the lens in children. This technique appears safe, effective, and easy to learn based on our preliminary results.

\section{Summary}

\section{What was known before}

- Spontaneous anterior dislocation of a crystalline lens is a rare entity. It requires immediate surgical intervention for optimal outcome. 


\section{What this study adds}

- Intralenticular lens aspiration is a safe and effective procedure for the management of anterior dislocated lens in paediatric cases. Microscope integrated OCT (MiOCT) may be helpful for cases with poor visibility.

\section{Compliance with ethical standards}

Conflict of interest The authors declare that they have no conflict of interest.

Publisher's note: Springer Nature remains neutral with regard to jurisdictional claims in published maps and institutional affiliations.

\section{References}

1. Vasavada AR, Praveen MR, Desai C. Management of bilateral anterior dislocation of a lens in a child with Marfan's syndrome. J Cataract Refract Surg. 2003;29:609-13.

2. Mukhopadhyaya U, Chakraborti C, Mondal A, Pattyanayak U, Agarwal RK, Tripathi P. Spontaneous dislocation of a crystalline lens to the anterior chamber with pupillary block glaucoma in Noonan Syndrome: a case report. Pan Afr Med J. 2014;17:135.

3. Ismail S, Khairy-Shamel S-T, Hussein A, Shaharuddin B, Embong Z, Ibrahim M. Spontaneous bilateral anterior lens dislocation in an 8-year-old girl. J Pediatr Ophthalmol Strabismus. 2010;47:111-3.

4. Ram J, Gupta N. Bilateral spontaneous anterior dislocation of crystalline lens in an infant. Lancet. 2011;378:1501.

5. Jovanović M. Bilateral spontaneous crystalline lens dislocation to the anterior chamber: a case report. Srp Arh Celok Lek. 2013;141:800-2.

6. Kim YJ, Ha SJ. Intracapsular lens extraction for the treatment of pupillary block glaucoma associated with anterior subluxation of the crystalline lens. COP. 2013;4:257-64.

7. Khokhar S, Aron N, Yadav N, Pillay G, Agarwal E. Modified technique of endocapsular lens aspiration for severely subluxated lenses. Eye. 2018;32:128-35.

8. Sinha R, Sharma N, Vajpayee RB. Intralenticular bimanual irrigation: aspiration for subluxated lens in Marfan's syndrome. J Cataract Refract Surg. 2005;31:1283-6.

9. Meredith TA, Maumenee AE. A review of one thousand cases of intracapsular cataract extraction: I. Complications. Ophthalmic Surg. 1979;10:32-41.

10. Lam A, Seck CM, Faye M, Brassier PK, Cisse MB, Diagne M. Spontaneous andbilateral anterior luxation of the lens in a 5-yearold child. J Fr Ophtalmol. 1996;19:279-82.

11. Chung JL, Kim SW, Kim JH, Kim TI, Lee HK, Kim EK. A case of Weill-Marchesanisyndrome with inversion of chromosome 15. Korean J Ophthalmol. 2007;21:255-60. 